\title{
Erratum to: A micro-computed tomography study of canal configuration of multiple-canalled mesiobuccal root of maxillary first molar
}

\author{
Yeun Kim • Seok-Woo Chang • Jong-Ki Lee • \\ I-Ping Chen • Blythe Kaufman • Jin Jiang • \\ Bruce Y. Cha • Qiang Zhu • Kamran E. Safavi • \\ Kee-Yeon Kum
}

Published online: 26 October 2012

(C) Springer-Verlag Berlin Heidelberg 2012

\section{Erratum to: Clin Oral Invest \\ DOI 10.1007/s00784-012-0852-8}

The original version of this article inadvertently contained a mistake.

There is a typo found in the Material and methods section. Please see below for the correct Vertucci classification.

- Vertucci type II (Weine type II). Two separate canals leave the pulp chamber and join short of the apex to form one canal.

- Vertucci type III. One canal leaves the pulp chamber, divides into two within the root, and then merges to exit as one canal.
- Vertucci type IV (Weine type III). Two separate and distinct canals extend from the pulp chamber to the apex.

- Vertucci type V (Weine type IV). One canal leaves the pulp chamber and divides short of the apex into two separate and distinct canals with separate apical foramina.

- Vertucci type VI. Two separate canals leave the pulp chamber, merge in the body of the root, and redivide short of the apex and exit as two distinct canals.

- Vertucci type VII. One canal leaves the pulp chamber, divides and then rejoins within the body of the root, and finally redivides into two distinct canals short of the apex.

- Vertucci type VIII. Three separate and distinct canals extend from the pulp chamber to the apex.

I.-P. Chen • B. Kaufman · J. Jiang • B. Y. Cha $\cdot$ Q. Zhu •

K. E. Safavi

Division of Endodontology, School of Dental Medicine,

University of Connecticut Health Center,

Farmington, CT, USA

K.-Y. Kum $(\bowtie)$

Jongro-Gu Yungun-Dong, Department of Conservative Dentistry, Dental Research Institute and BK 21 Program, School of Dentistry,

Seoul National University,

Seoul, Republic of Korea

e-mail: kum6139@snu.ac.kr 